\title{
Demineralized Bone Matrix, as a Graft Enhancer of Auto-Local Bone in Posterior Lumbar Interbody Fusion
}

\author{
Dong Ki Ahn, Sang Ho Moon, Tae Woo Kim, Kyung Hwan Boo, Sung Won Hong \\ Department of Orthopedic Surgery, Seoul Sacred Heart General Hospital, Seoul, Korea
}

\begin{abstract}
Study Design: A case controlled study with prospective data collection.
Purpose: To evaluate the early influence and the final consequence of demineralized bone matrix (DBM) on auto-local bone as a graft enhancer in posterior lumbar interbody fusion (PLIF).

Overview of Literature: DBM is known as an osteoinductive material; however, it has not been clearly recognized to enhance autolocal bone with a small amount.

Methods: Patients who had a PLIF were allocated into two groups. Group I (70 cases) used auto-local bone chips and group II (44 cases) used DBM as an additive to auto-local bone, $1 \mathrm{~mL}$ per a segment. Group selection was alternated. Early assessment was performed by computed tomography at 6 months and final assessment was done by simple radiography after 24 months at least. The degree of bone formation was assessed by 4 grade scale.

Results: The subjects of both groups were homogenous and had similar Oswestry Disability Index at final assessment. The ratio of auto-local bone chips and DBM was 6:1. The degree of bone formation at 6 months after surgery was superior in group II. However, there was no significant difference between the two groups at the final assessment.

Conclusions: DBM was not recognized to enhance auto-local bone with small amount.
\end{abstract}

Keywords: Demineralized bone matrix; Graft enhancer; Posterior lumbar interbody fusion

\section{Introduction}

Is it helpful to add petit demineralized bone matrix (DBM) for an instrumented posterior lumbar interbody fusion (PLIF) with auto-local bone?

Arthrodesis of the spine has been used to treat various sorts of spinal disease and more than $50 \%$ of bone graft has been used for that purpose $[1,2]$. Success rate of spinal fusion has been raised up as instrumentation has developed. Increment of patient's satisfaction, however, has been limited significantly due to the morbidities of autograft donor site. There are several options that have been used independently or as a combination to avert the autoiliac bone harvesting. Those were as follows; allogeneic bone graft, auto-local bone graft, bone substitute graft, interbody bone graft, increasing fixation power by pedicle screw system and cages. DBM, which enhances osteoinductibity, was appraised available as a graft extender of the auto- iliac bone in large volume. However, its value was skeptical as an adjunct to the auto-local bone or other

Received Nov 29, 2012; Revised Apr 15, 2013; Accepted May 10, 2013

Corresponding author: Dong Ki Ahn

Department of Orthopedic Surgery, Seoul Sacred Heart General Hospital,

259 Wangsan-ro, Dongdaemun-gu, Seoul 130-867, Korea

Tel: +82-2-968-2394, Tel: +82-2-966-1616, E-mail: adkajs@hanmail.net 
ostoeconductive materials. DBM is recognized as an osteoinductive material; hence, it should be meant to act as a graft enhancer for the graft materials, which have a litter or no osteoinductivity. As PLIF technique has been becoming popular, auto-local bone chips fortified by DBM have been used as a graft material more and more. However, there has been no clinical study that supports the availability of such construct thus far. To my knowledge, PLIF using autolocal bone chips and small amount of DBM is regarded as a best sample to appraise this subject. Furthermore, in case of instrumented PLIF with pedicle screw and cage system, structural support can be fortified and an interbody space, which is surrounded by large cancellous plates, would give more favorable circumstance than posteolateral fusion (PLF) for osteosynthesis. There have been many reports that note successful fusion rate in this sort of spinal fusion surgery even without an annexing of DBM. Therefore, we designed two stage investigations to see the early influence and the final consequence of DBM on auto-local bone as a graft enhancer. As the first stage, bone formation status was evaluated at 6 months after the index surgeries, which has been noted as a usual fusion time after spine surgery and as the second stage final fusion rate was evaluated at least 2 years after the index surgeries.

\section{Materials and Methods}

\section{Materials}

It was designed as a case controlled study; however, data were collected prospectively. The patients of degenerative spinal disease or spondylolytic spondylolysthisis, who underwent spinal fusion surgeries between January 2006 and October 2006, were included. Those who under 50 and over 80 years old, more than 3 segments and more than 3 times of surgery were excluded. A total of 114 cases of 92 patients underwent surgeries during the index period. Seventy cases of group I used auto-local bone chips and 44 cases of group II used auto-local bone chips and $1 \mathrm{~mL}$ of DBM per segment. The type of bone graft was alternated according to the date and previous consent was obtained in group II cases. If a patient refused to use DBM, he or she was shifted to group I.

\section{Method of surgery}

Conventional posterior approach was used. Decompres- sion was performed unilaterally or bilaterally according to the necessity. Disc extirpation was performed unilaterally in all cases. Pedicle screws and rods were instrumented and a cage per a segment was inserted through a symptom dominant side. The auto-local bones that were achieved while performing decompression were made into small chips of 1 to $2 \mathrm{~mm}$. In group I, those were grafted into the interbody space and a cage that was charged with them was inserted at one side. In group II, $1 \mathrm{~mL}$ of DBM per segment was annexed to auto-local bone chips and grafted

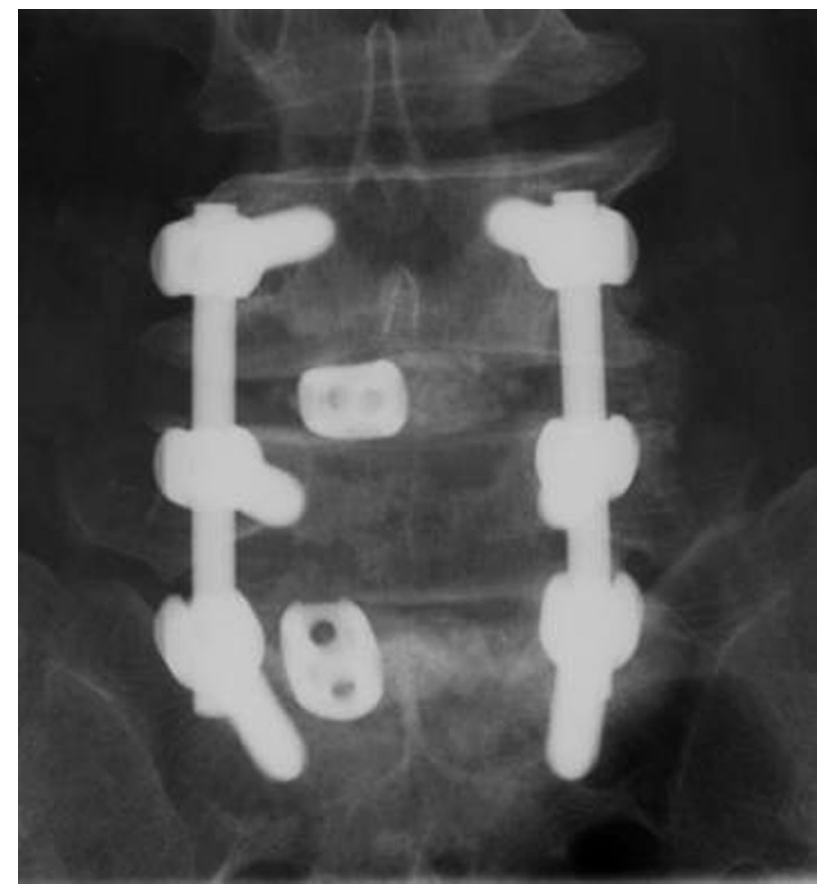

Fig. 1. We performed posterolateral interbody fusion using unilateral cage. In goup I, the only grafted materials were auto-local bone chips, but in group II $1 \mathrm{~mL}$ of demineralized bone matrix (Allomatrix) per a segment was annexed to auto-local bone chips.

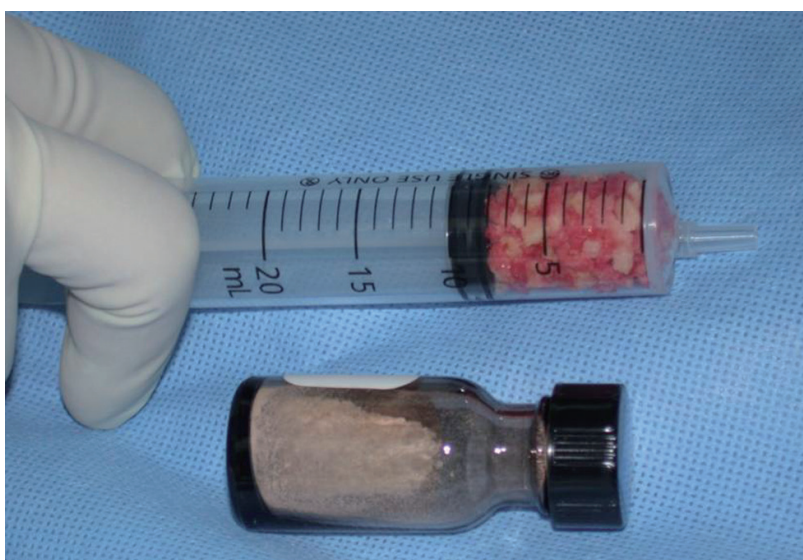

Fig. 2. The amount of grafted auto-local bone chips was measured by a $20 \mathrm{~mL}$ syringe in the maximum compression state. 
with the same manner as group I (Fig. 1). All cases used Allomatrix (Wright Medical, Arlington, TN, USA). The amount of auto-local bone chips was measured by a 20 $\mathrm{mL}$ syringe in the maximum compression state (Fig. 2).

\section{Method of analysis}

Sampling homogeneity of the two groups was reviewed in terms of age, sex, drinking, smoking, medical co- morbidity, such as diabetes mellitus, and other endocrine diseases, previous surgeries at the same segments, bone mineral density, amount of grafted bone per segment and functional score by Oswestry Disability Index ver. 2 (ODI). The norm of frequent alcohol drinker was more than twice a week and more than 1 year before surgery. Bone mineral density was examined at the femur neck by Dual-energy X-ray absorptiometry (Osteoprima, MediRay, Suwon, Korea). Preoperative and final clinical

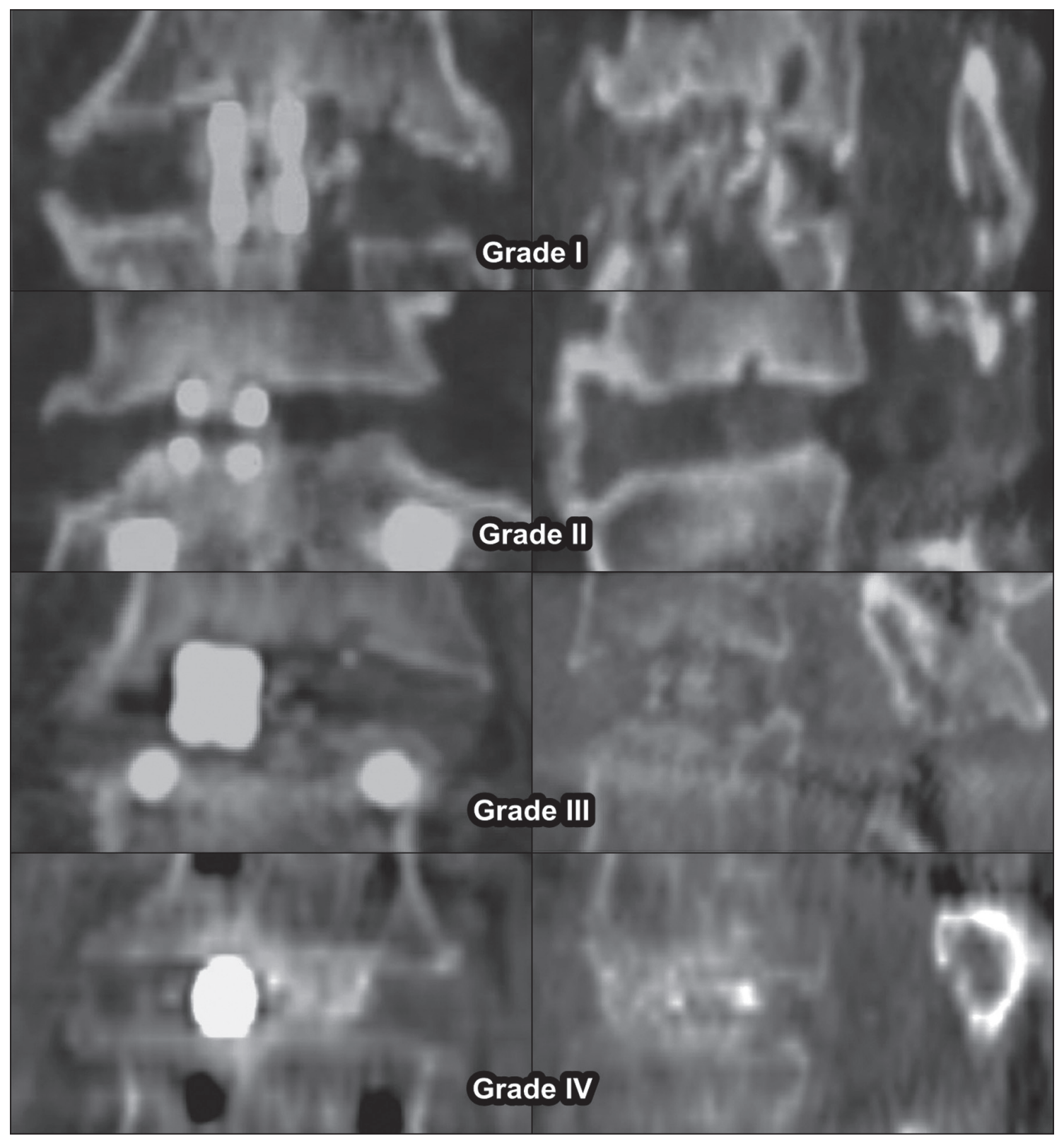

Fig. 3. At 6 month follow-up periods, mid-sagittal image and anterior 1/3 coronal computed tomography images of $19 \%$ of group I and $43 \%$ of group II were assessed by the author's own method; grade I is almost no bone formation, grade II is bone formation of less than $50 \%$ of grated zone, grade III is bone formation of more than $50 \%$ but incomplete continuity of bone mass and grade IV is diffuse and continuous bone formation. 
functional status was assessed by ODI. Complications, especially osteoinductive material induced complications, were investigated.

1) Early assessment by computed tomogram

The degree of bone formation was assessed by sagittal and coronal reconstruction images at 6 months \pm 2 weeks. Thirty two cases of 27 patients were examined during the pertinent period. Group I was 13 cases (19\%) and group II was 19 cases (43\%). Mid-sagittal image and anterior $1 / 3$ coronal image were assessed by the method devised by the senior author (Fig. 3). The details were as follows; grade I is almost no bone formation, grade II is bone formation of less than $50 \%$ of grafted zone, grade III is bone formation of more than $50 \%$, but incomplete continuity of bone mass and grade IV is diffuse and continuous bone formation. Numeric scales of 1 to 4 were given to each grade. Two orthopedic doctors, who were blind to the study design read the images independently. The sum of each plane scores of the two readers was counted as a final score for the segment. Each segment was counted as a case.

\section{2) Final assessment by plain radiography}

The minimum follow-up was limited to 24 months. A total of 99 cases of 79 patients were legitimate to this norm. Group I was 61 cases (87\%) and group II was 38 cases (86\%) (Fig. 4). Degree of bone formation was assessed on the final sagittal plain radiography by the 4 grade numeric scale method devised by the senior author. Grade I is no or little bone formation, grade II is bone formation less than $50 \%$ of the grafted zone, grade III is bone formation more than $50 \%$ of the grafted zone and grade IV is full bone formation of the interbody space, which connects both end plates (Fig. 5). The readers were the same orthopedic doctors with the same condition and same manner as the 6 month computed tomography (CT) assessment. A statistical analysis was conducted by $t$-test, Fisher's exact test and Likelihood ratio method for the assessment of sampling homogeneity, Kappa test for the concordance between the two readers and Mann-Whitney test for the difference between the two groups. SPSS ver. 16.0 package (SPSS Inc., Chicago, IL, USA) was used.

\section{Results}

The demography of the two groups was not heterogeneous (Table 1). The amount ratio of local bone chips and DBM was 6.0:1 in group II. The mean follow up period was 34 months (range, 24-51 months). Surgery related complications and DBM related complications did not develop in both groups. ODI of both groups were improved significantly at the final assessment and there was no difference between the two groups (Table 2). There was a moderate degree concordance between the two readers in sagittal (kappa $=0.494, p<0.001)$ and coronal

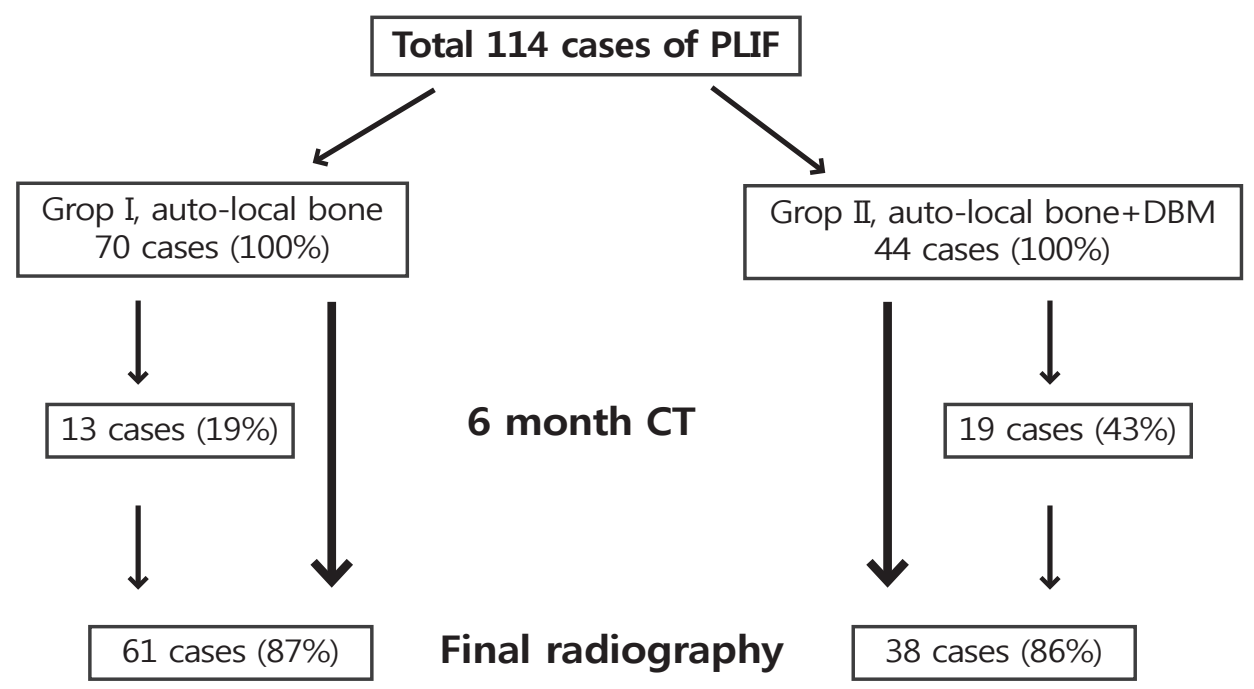

Fig. 4. A diagram of how many cases were included in the radiological assessments. PLIF, posterior lumbar interbody fusion; DBM, demineralized bone matrix; CT, computed tomography. 
(kappa $=0.467, p<0.001)$ CT images in preliminary assessment and plain sagittal radiography (kappa $=0.57$, $p=0.000)$ in the final assessment.

Bone formation at 6 months after surgery was superior in group II. However, there was no significant difference between the two groups at the final assessment (Table 3).

\section{Discussion}

The use of bone graft substitutes in spine surgery has been researched and debated. Although auto-iliac bone remains as a gold standard, its potential complications have led to the development of other bone graft options. DBM has been used as one of the alternatives of the autoiliac bone. DBM has demonstrated an effect on the differentiation of osteoprogenitor cells into osteoblast. Urist [3] first identified an osteoinductive substance, while preparing extracts from the demineralized bone. Since then, the osteoinductivity of DBM has been well established [46]. DBM is mainly comprised of type I collagen (93\%),
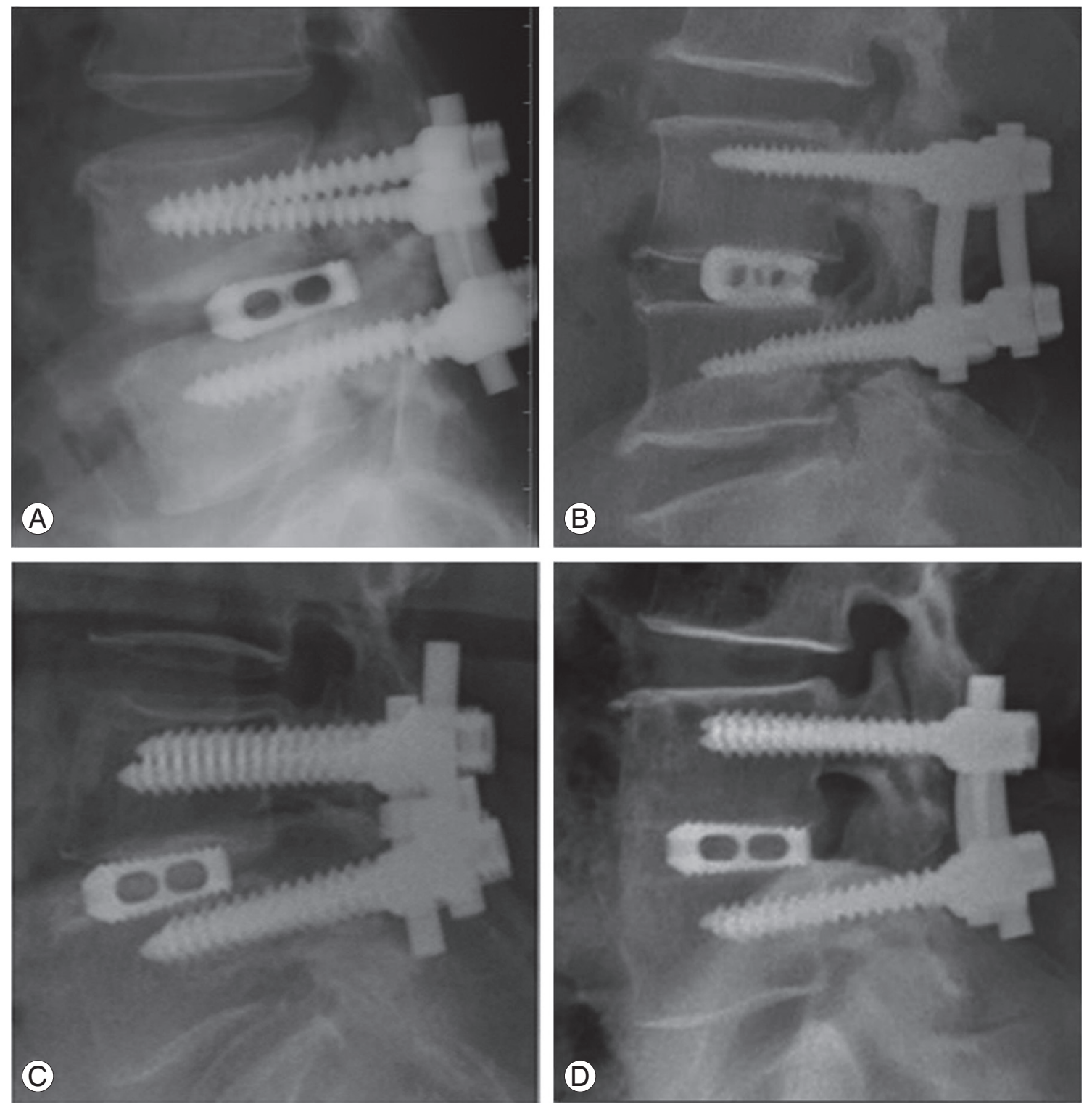

Fig. 5. At 24 month follow-up periods, Degree of bone formation was assessed by the final sagittal plain radiography on the basis of author's own method. (A) Grade I is no or little bone formation at the graft zone, (B) grade II is bone formation less than $50 \%$ of the graft zone, (C) grade III is bone formation more than $50 \%$ of the graft zone, (D) grade IV is full bone formation of the interbody space which connects the both end plates. 
Table 1. Homogeneity of two groups

\begin{tabular}{|c|c|c|c|}
\hline Characteristic & Group I & Group II & $p$-value \\
\hline Age (yr) & $63.7 \pm 6.5$ & $64.5 \pm 5.5$ & 0.548 \\
\hline $\operatorname{Sex}(M / F)$ & $14 / 45$ & $4 / 34$ & 0.090 \\
\hline Drinking & $10 / 61$ & $4 / 38$ & 0.557 \\
\hline Smoking & $16 / 61$ & $3 / 38$ & 0.639 \\
\hline Diabetes mellitus & $21 / 61$ & $12 / 38$ & 0.770 \\
\hline Other endocrine dis. & $6 / 61$ & $4 / 38$ & 0.912 \\
\hline Previous surgeries & $6 / 61$ & $7 / 38$ & 0.225 \\
\hline Bone mineral density (femur neck) & $-1.9 \pm 0.9$ & $-1.8 \pm 1.1$ & 0.545 \\
\hline Amount of local bone (mL) & $6.1 \pm 1.2$ & $6.0 \pm 1.0$ & 0.775 \\
\hline Preoperative Oswestry Disability Index & $36.5 \pm 7.7$ & $37.7 \pm 8.0$ & 0.440 \\
\hline
\end{tabular}

Table 2. Functional improvement of two groups

\begin{tabular}{lccc}
\multicolumn{1}{r}{ Characteristic } & Preoperative ODI & Final 0DI & $p$-value \\
Group I (\%) & $63.9 \pm 10.0$ & $36.5 \pm 7.7$ & 0.000 \\
Group II (\%) & $61.8 \pm 12.7$ & $37.4 \pm 8.0$ & 0.000 \\
\hline -value & 0.400 & 0.440 & - \\
\hline
\end{tabular}

ODI, Oswestry Disability Index.

Table 3. Degree of bone formation of two groups

\begin{tabular}{lccc}
\multicolumn{1}{c}{ Characteristic } & Group I & Group II & $p$-value \\
\hline 6 month CT/full score & $10.4 \pm 2.1 / 16$ & $6.9 \pm 3.0 / 16$ & 0.002 \\
Final radiography/full score & $6.7 \pm 1.4 / 8$ & $6.6 \pm 1.1 / 8$ & 0.768 \\
\hline
\end{tabular}

CT, computed tomography.

which provides an osteoconductive surface. Noncollagenous soluble proteins, such as osteoinductive bone morphogenic protsein (BMP) and a cocktail of synergistic proteins (transforming growth factor-beta, insulin-like growth factor, platelet-derived growth factor, fibroblast growth factor) represent $5 \%$. The remaining $2 \%$ is made of residual mineralized matrix [7-10]. In addition to its osteoinductivity, DBM has some degree of osteoconductivity [5].

DBM is combined with other components, so called carrier, intended to make easier to handle and enhance localization. The carrier must be biocompatible with the bone, maintain graft localization and not reduce the osteoconductivity [11]. The first DBM/carrier products were introduced in 1991 and have since become one of the most widely used alternative graft in spine fusion surgery. Even though there are many kinds of growth factor proteins, the osteoinductive ability of DBM largely depends on the activity of BMP [12]. Unfortunately, the production of DBM is loosely regulated by Food and Drug Administration as a minimally manipulated human allograft tissue, with no mandated requirements for the osteogenic growth factors concentration or for the demonstration of osteogenic efficacy. The content of $\mathrm{BMP}$ is variable according to the manufacturers and even the different lots of the same manufacturer have variable amounts of BMP. BMP-2 and BMP-7 exist in nanogram concentrations in DBM, which is 1 million times less than the concentration, which is required to produce a lumbar spinal fusion [13]. Herein, it is unavoidable that the amount of osteogenic activity of a particular DBM is highly dependent upon the donor variability [14]. Commercially available DBMs have demonstrated the variability of their osteoinductive potential, which may 
reflect differences of their BMP content [15]. To avoid variability between the different commercial products, only one product, Allomatrix (Wright Medical) was used in the current study, which is comprised of $86 \%$ of DBM and $14 \%$ of calcium sulfate hemihydrates as a carrier and electron beam was used for final sterilization.

There have been many animal studies that presented promising results. Several rat studies have demonstrated that DBM can induce a spinal fusion in a dose dependent manner and may have value as a graft substitute, at least as a graft extender $[11,15,16]$. However, the substances did not perform equally, that some did not demonstrate any significant bone formation. There is also a rat study which noted that DBM is superior to the fresh frozen allogeneic bone in arthrodesis [17]. In a canine study, combination of DBM with autograft achieved more rapid spinal fusion than the autograft alone [6], and in rabit interbody fusion model, composite graft of DBM and hydroxyapatite block showed more rapid and strong fusion than autograft alone [18]. In a study which used more advanced species -nonhuman primate- admitted DBM as a graft enhancer [19].

Despite promising animal data, DBM has been under a lack significant clinical data that support its efficacy in spine fusion surgeries so far. The initial human study on the efficacy of DBM was performed on the anterior cervical spine fusion in which the freeze-dried allogeneic bone augmented by DBM was compared to the auto-iliac bone. This study did not offer sufficient osteoinductivity of the allograft-DBM construct in anterior cervical fusion [20]. There is a study somewhat encouraging, which compared the fusion rates of auto-local bone and DBM constructs to that of the auto-iliac bone alone cases in the setting of PLF. The result that presented no difference between the two groups indicated that it has a value as a graft extender in humans [21].

We performed the current study to verify the graft enhancing ability of DBM. It was considered that a substance that has osteoinductivity should be able to act as a graft enhancer rather than a graft extender. The construct of PLIF with pedicle screws and cages supplies sufficient stability to reduce the contribution of the graft materials. There have been many studies which showed satisfactory fusion rate with local bone chips in PLIF using pedicle screws and cages. Given our own experience, however, nonunion cases have been developed even with such construct. There is a study that said the necessity of an additional graft adjuvant to increase fusion quality and prevent subsidence in the same kind of surgeries [22].

There have been several reports as to the efficacy of BMP and auto-local bone composite graft in TLIF or PLIF [23,24]. Meanwhile, there also have been many studies that noted complications, such as ectopic bone formation [25], radiculitis [26] and vertebral osteolysis [27], which are attributable to BMP in PLIF. To our knowledge, there have been a few reports which noted the graft enhancing effect and complications of DBM when it is used in PLIF with the local chip bone. We thought that PLIF using pedicle screws and unilateral cage augmented with local chip bone is a good model to evaluate the efficacy of DBM as a graft enhancer because that kind of surgery is already proven to have a high fusion rate; therefore, it would be least detrimental even though DBM does not work and the space opposite to a cage would provide good visualization to appraise the quantity of fusion mass. Furthermore, large cancellous surfaces of the vertebral end plates were presumed to act as a instant source of osteogenic precursor cells as mentioned by Bauer and Muschler [28]. According to the study of Wang and Gilmcher [29], the dominant pathway of DBM osteogenesis is akin to endochondral bone formation in the subcutaneous and intramuscular layer; whereas, it resembles intramembranous bone formation in the cranial defect. The authors have suggested that this distinction may result from the mesenchymal stem cells in different position having a predominance of stem cells with different receptors that selectively bind chondrogenic or osteogenic proteins, respectively. The intervertebral space was considered to resemble to a cranial defect; therefore, DBM was expected to work in a more potent way than the intertransverse plane. We applied the 4 grade quantification scales rather than a dichotomous fusion or nonunion to help discriminate the contribution of DBM more delicately.

At first, we planned to assess whether they are fused or not with a simple radiography at 6 months and last follow-up. It was, however, totally impossible to be decided at 6 months. Nearly all cases of both groups were classified as nonunion, same or less than grade II. Therefore, the early assessment was changed to be based on CT findings. Because of the above reason and time constraint (within the time 6 months \pm 2 weeks), CT follow-up rate became significantly lower. For that reason, the result of early assessment was considered to have a meager signifi- 
cance, though it showed early bone formation.

One of the drawbacks of DBM is inconsistent amount of BMP content $[13,15]$. We expected superior score in group II, despite the large standard deviation. However, the results showed similar average score and standard deviation. Therefore, we concluded that it does not indicate inconsistent osteoinductivity, but unwarrantable osteoinductivity. Sassard et al. [21] reported that DBM acted as a graft extender when it was mixed with auto-iliac bone in 3:1 ratio. In my opinion, that cannot be as an osteoinductive material. It is rather close to osteoconduction, which is attributable to a large amount of collagen and carrier materials.

There are several limitations in the current study. The sample size of the two groups was not similar because those who refused to use DBM were transferred to the opposite group. The follow-up rate of preliminary CT assessment at 6 months was too low to assert a certain conclusion due to the above mentioned reasons. The concordance between the two readers was not strong enough.

\section{Conclusions}

DBM did not present a graft enhancing effect when small amount of it was used as a composite graft with the autolocal bone in PLIF using pedicle screws and cages. The early bone forming effect of DBM was not conclusive due to the significant flaw in the early stage assessment. Therefore, DBM is untenable as a graft enhancer universally.

\section{Conflict of Interest}

No potential conflict of interest relevant to this article was reported.

\section{References}

1. Lee KJ, Roper JG, Wang JC. Demineralized bone matrix and spinal arthrodesis. Spine J 2005;5(6 Suppl):217S-23S.

2. Vaccaro AR, Chiba K, Heller JG, et al. Bone grafting alternatives in spinal surgery. Spine J 2002;2:206-15.

3. Urist MR. Bone: formation by autoinduction. Science 1965;150:893-9.

4. Berven S, Tay BK, Kleinstueck FS, Bradford DS. Clinical applications of bone graft substitutes in spine surgery: consideration of mineralized and demineralized preparations and growth factor supplementation. Eur Spine J 2001;10 Suppl 2:S169-77.

5. Martin GJ Jr, Boden SD, Titus L, Scarborough NL. New formulations of demineralized bone matrix as a more effective graft alternative in experimental posterolateral lumbar spine arthrodesis. Spine (Phila Pa 1976) 1999;24:637-45.

6. Frenkel SR, Moskovich R, Spivak J, Zhang ZH, Prewett AB. Demineralized bone matrix. Enhancement of spinal fusion. Spine (Phila Pa 1976) 1993;18:1634-9.

7. Linkhart TA, Mohan S, Baylink DJ. Growth factors for bone growth and repair: IGF, TGF beta and BMP. Bone 1996;19(1 Suppl):1S-12S.

8. Massague J. The transforming growth factor-beta family. Annu Rev Cell Biol 1990;6:597-641.

9. Jingushi S, Heydemann A, Kana SK, Macey LR, Bolander ME. Acidic fibroblast growth factor (aFGF) injection stimulates cartilage enlargement and inhibits cartilage gene expression in rat fracture healing. J Orthop Res 1990;8:364-71.

10. Aspenberg P, Albrektsson T, Thorngren KG. Local application of growth-factor IGF-1 to healing bone. Experiments with a titanium chamber in rabbits. Acta Orthop Scand 1989;60:607-10.

11. Lee YP, Jo M, Luna M, Chien B, Lieberman JR, Wang JC. The efficacy of different commercially available demineralized bone matrix substances in an athymic rat model. J Spinal Disord Tech 2005;18:439-44.

12. Miyazaki M, Tsumura H, Wang JC, Alanay A. An update on bone substitutes for spinal fusion. Eur Spine J 2009;18:783-99.

13. Bae HW, Zhao L, Kanim LE, Wong P, Delamarter $\mathrm{RB}$, Dawson EG. Intervariability and intravariability of bone morphogenetic proteins in commercially available demineralized bone matrix products. Spine (Phila Pa 1976) 2006;31:1299-306.

14. Russell JL, Block JE. Clinical utility of demineralized bone matrix for osseous defects, arthrodesis, and reconstruction: impact of processing techniques and study methodology. Orthopedics 1999;22:524-31.

15. Wang JC, Alanay A, Mark D, et al. A comparison of commercially available demineralized bone matrix for spinal fusion. Eur Spine J 2007;16:1233-40.

16. Qiu QQ, Shih MS, Stock K, et al. Evaluation of DBM/ AM composite as a graft substitute for posterolateral 
lumbar fusion. J Biomed Mater Res B Appl Biomater 2007;82:239-45.

17. Oikarinen J. Experimental spinal fusion with decalcified bone matrix and deep-frozen allogeneic bone in rabbits. Clin Orthop Relat Res 1982;(162):210-8.

18. Ragni P, Lindholm TS. Interaction of allogeneic demineralized bone matrix and porous hydroxyapatite bioceramics in lumbar interbody fusion in rabbits. Clin Orthop Relat Res 1991;(272):292-9.

19. Louis-Ugbo J, Murakami H, Kim HS, Minamide A, Boden SD. Evidence of osteoinduction by Grafton demineralized bone matrix in nonhuman primate spinal fusion. Spine (Phila Pa 1976) 2004;29:360-6.

20. An HS, Simpson JM, Glover JM, Stephany J. Comparison between allograft plus demineralized bone matrix versus autograft in anterior cervical fusion. A prospective multicenter study. Spine (Phila Pa 1976) 1995;20:2211-6.

21. Sassard WR, Eidman DK, Gray PM, et al. Augmenting local bone with Grafton demineralized bone matrix for posterolateral lumbar spine fusion: avoiding second site autologous bone harvest. Orthopedics 2000;23:1059-64.

22. Lee JH, Jeon DW, Lee SJ, Chang BS, Lee CK. Fusion rates and subsidence of morselized local bone grafted in titanium cages in posterior lumbar interbody fusion using quantitative three-dimensional computed tomography scans. Spine (Phila Pa 1976) 2010;35:1460-5.

23. Villavicencio AT, Burneikiene S, Nelson EL, Bulsara KR, Favors M, Thramann J. Safety of transforaminal lumbar interbody fusion and intervertebral recombinant human bone morphogenetic protein-2. J Neurosurg Spine 2005;3:436-43.

24. Mummaneni PV, Pan J, Haid RW, Rodts GE. Contribution of recombinant human bone morphogenetic protein-2 to the rapid creation of interbody fusion when used in transforaminal lumbar interbody fusion: a preliminary report. Invited submission from the Joint Section Meeting on Disorders of the Spine and Peripheral Nerves, March 2004. J Neurosurg Spine 2004;1:19-23.

25. Haid RW Jr, Branch CL Jr, Alexander JT, Burkus JK. Posterior lumbar interbody fusion using recombinant human bone morphogenetic protein type 2 with cylindrical interbody cages. Spine J 2004;4:527-38.

26. Rihn JA, Patel R, Makda J, et al. Complications associated with single-level transforaminal lumbar interbody fusion. Spine J 2009;9:623-9.

27. Lewandrowski KU, Nanson C, Calderon R. Vertebral osteolysis after posterior interbody lumbar fusion with recombinant human bone morphogenetic protein 2: a report of five cases. Spine J 2007;7:609-14.

28. Bauer TW, Muschler GF. Bone graft materials. An overview of the basic science. Clin Orthop Relat Res 2000;(371):10-27.

29. Wang J, Gilmcher MJ. Characterization of matrixinduced osteogenesis in rat calvarial defects: I. Differences in the cellular response to demineralized bone matrix implanted in calvarial defects and in subcutaneous sites. Calcif Tissue Int 1999;65:156-65. 